\title{
Hammett Correlation of Nornicotine Analogs in the Aqueous Aldol Reaction: Implications for Green Organocatalysis
}

\section{Supporting Information}

Claude J. Rogers, Tobin J. Dickerson, Andrew P. Brogan, and Kim D. Janda*

Department of Chemistry and The Skaggs Institute for Chemical Biology

The Scripps Research Institute

10550 North Torrey Pines Road

La Jolla, CA 92037 USA

Telephone: +18587842516

Fax: +1 8587842595

Email:kdjanda@scripps.edu

Table of Contents:

General Methods S2

${ }^{1} \mathrm{H}$ NMR and ${ }^{13} \mathrm{C}$ NMR Peak Listings of 2-Arylpyrrolidines $\mathbf{1 b - 1 0 b}$

${ }^{1} \mathrm{H}$ NMR and ${ }^{13} \mathrm{C}$ NMR Spectra of 2-Arylpyrrolidines $\mathbf{4 b - 1 0 b}$

S5-S18 
General methods. All reactions were conducted in oven-dried glassware under an atmosphere of Ar. Hexanes, $\mathrm{CH}_{2} \mathrm{Cl}_{2}$, and $\mathrm{Et}_{3} \mathrm{~N}$ were distilled from $\mathrm{CaH}_{2}$, and THF from $\mathrm{Na} /$ benzophenone. 1-(3-bromopropyl)-2,2,5,5-tetrametyl-1-aza-2,5-disilacyclopentane was distilled prior to use. Unless otherwise stated, all other commercial reagents were used without further purification. ${ }^{1} \mathrm{H}$ and ${ }^{13} \mathrm{C}$-NMR were acquired at 400, 500, and 600 $\mathrm{MHz}$ respectively for ${ }^{1} \mathrm{H}$ and 100,125 , and $150 \mathrm{MHz}$ respectively for ${ }^{13} \mathrm{C}$. All analytical reverse-phase HPLC were preformed on a $\mathrm{C}_{18}$ reverse-phase column with an isocratic mobile phase of $25 \% \mathrm{MeCN}, 75 \% \mathrm{H}_{2} \mathrm{O}$ containing $0.1 \%$ TFA, a $1.0 \mathrm{~mL} \mathrm{~min}^{-1}$ flow rate, and detection at $254 \mathrm{~nm}$. HPLC solvents were filtered and degassed prior to use.

2-[4-(N,N-Dimethylamino)phenyl]pyrrolidine (1b). ${ }^{1}{ }^{1} \mathrm{H}-\mathrm{NMR}\left(500 \mathrm{MHz}, \mathrm{CDCl}_{3}\right)$ $\delta 7.22(\mathrm{~d}, J=8.5 \mathrm{~Hz}, 2 \mathrm{H}), 6.70(\mathrm{~d}, J=8.5 \mathrm{~Hz}, 2 \mathrm{H}), 4.00(\mathrm{t}, J=8 \mathrm{~Hz}, 1 \mathrm{H}), 3.18$ (ddd, $J=$ $10 \mathrm{~Hz}, 8 \mathrm{~Hz}, 5 \mathrm{~Hz}, 1 \mathrm{H}), 2.98-2.91$ (m, 7H), 2.14-2.07 (m, 1H), 1.94-1.78 (m, 2H), 1.68$1.61(\mathrm{~m}, 1 \mathrm{H}) .{ }^{13} \mathrm{C}-\mathrm{NMR}\left(100 \mathrm{MHz}, \mathrm{CDCl}_{3}\right) \delta$ 149.7, 127.4, 125.6, 112.7, 62.3, 46.7, 40.75, 33.9, 25.5. ESI-TOF calc. $\mathrm{C}_{12} \mathrm{H}_{18} \mathrm{~N}_{2}\left[\mathrm{M}+\mathrm{H}^{+}\right]$191.1543, found 191.1538 .

2-(4-Methoxyphenyl)pyrrolidine (2b). ${ }^{1} \mathrm{H}-\mathrm{NMR}\left(500 \mathrm{MHz}, \mathrm{CDCl}_{3}\right) \delta 7.28(\mathrm{~d}, J=8.5$ $\mathrm{Hz}, 2 \mathrm{H}), 6.86(\mathrm{~d}, J=8.5 \mathrm{~Hz}, 2 \mathrm{H}), 4.05(\mathrm{t}, J=8 \mathrm{~Hz}, 1 \mathrm{H}), 3.80$ (s, 3H), 3.20 (ddd, $J=10$ Hz, 7.5 Hz, $5 \mathrm{~Hz}, 1 \mathrm{H}), 2.99$ (ddd, $J=10 \mathrm{~Hz}, 8 \mathrm{~Hz}, 6.5 \mathrm{~Hz}, 1 \mathrm{H}), 2.18-2.12(\mathrm{~m}, 1 \mathrm{H}), 1.96-$ $1.81(\mathrm{~m}, 2 \mathrm{H}), 1.68-1.61(\mathrm{~m}, 1 \mathrm{H}) .{ }^{13} \mathrm{C}-\mathrm{NMR}\left(125 \mathrm{MHz}, \mathrm{CDCl}_{3}\right) \delta 158.4,136.6,127.5$, 113.6, 62.0, 55.1, 46.9, 34.1, 25.4. ESI-TOF calc. $\mathrm{C}_{11} \mathrm{H}_{15} \mathrm{NO}\left[\mathrm{M}+\mathrm{H}^{+}\right] 178.1226$, found 178.1225.

2-Phenylpyrrolidine (3b). ${ }^{1} \mathrm{H}-\mathrm{NMR}\left(500 \mathrm{MHz}, \mathrm{CDCl}_{3}\right) \delta$ 7.38-7.30 (m, 4H), 7.23 (tt, $J=6.5 \mathrm{~Hz}, 1.5 \mathrm{~Hz}, 1 \mathrm{H}), 4.12(\mathrm{t}, J=7.5 \mathrm{~Hz}, 1 \mathrm{H}), 3.21(\mathrm{ddd}, J=10 \mathrm{~Hz}, 7.5 \mathrm{~Hz}, 5 \mathrm{~Hz}, 1 \mathrm{H})$,

\footnotetext{
${ }^{1}$ Yus, M.; Soler, T.; Foubelo, F. J. Org. Chem. 2001, 66, 6207-6208.
} 
$3.02(\mathrm{ddd}, J=10 \mathrm{~Hz}, 8 \mathrm{~Hz}, 6.5 \mathrm{~Hz}, 1 \mathrm{H}), 2.23-2.16(\mathrm{~m}, 1 \mathrm{H}), 1.98-1.82(\mathrm{~m}, 2 \mathrm{H}), 1.72-1.64$ (m, 1H). ${ }^{13} \mathrm{C}-\mathrm{NMR}(100 \mathrm{MHz}, \mathrm{CDCl} 3)$ 144.7, 128.3, 126.8, 126.5, 62.1, 47.0, 34.5, 25.4. ESI-TOF calc. $\mathrm{C}_{10} \mathrm{H}_{13} \mathrm{~N}\left[\mathrm{M}+\mathrm{H}^{+}\right]$148.1121, found 148.1121 .

2-(4-Fluorophenyl)pyrrolidine (4b). ${ }^{1} \mathrm{H}-\mathrm{NMR}\left(500 \mathrm{MHz}, \mathrm{CDCl}_{3}\right) \delta 7.33$ (dd, $J=8.5$ $\mathrm{Hz}, 5.5 \mathrm{~Hz}, 2 \mathrm{H}), 6.99$ (t, $J=8.5 \mathrm{~Hz}, 2 \mathrm{H}), 4.10(\mathrm{t}, J=7.5 \mathrm{~Hz}, 1 \mathrm{H}), 3.19$ (ddd, $J=10 \mathrm{~Hz}$, $7.5 \mathrm{~Hz}, 5 \mathrm{~Hz}, 1 \mathrm{H}$ ), 3.02 (ddd, $J=10 \mathrm{~Hz}, 8 \mathrm{~Hz}, 6.5 \mathrm{~Hz}, 1 \mathrm{H}$ ), 2.26-2.14 (m, 1H), 1.98-1.82 (m, 2H), 1.69-1.61 (m, 1H). ${ }^{13} \mathrm{C}-\mathrm{NMR}\left(100 \mathrm{MHz}, \mathrm{CDCl}_{3}\right) \delta$ 128.1, 128.0, 115.2, 115.0, 61.9, 46.7, 34.3, 25.4. ESI-TOF calc. $\mathrm{C}_{10} \mathrm{H}_{12} \mathrm{FN}\left[\mathrm{M}+\mathrm{H}^{+}\right]$166.1026, found 166.1026.

2-(3-Methoxyphenyl)pyrrolidine (5b). ${ }^{1} \mathrm{H}-\mathrm{NMR}\left(500 \mathrm{MHz}, \mathrm{CDCl}_{3}\right) \delta 7.23(\mathrm{t}, J=8$ Hz, 1H), $7.23(\mathrm{~m}, 2 \mathrm{H}), 6.77(\mathrm{dd}, J=8 \mathrm{~Hz}, 2.5 \mathrm{~Hz}, 1 \mathrm{H}), 4.10(\mathrm{t}, J=7.5 \mathrm{~Hz}, 1 \mathrm{H}), 3.81(\mathrm{~s}$, 3H), $3.20(\mathrm{ddd}, J=10 \mathrm{~Hz}, 7.5 \mathrm{~Hz}, 5 \mathrm{~Hz}, 1 \mathrm{H}), 3.01$ (ddd, $J=10 \mathrm{~Hz}, 8 \mathrm{~Hz}, 6.5 \mathrm{~Hz}, 1 \mathrm{H}$ ), 2.22-2.15 (m, 1H), 1.96-1.80 (m, 2H), 1.71-1.64 (m, 1H). ${ }^{13} \mathrm{C}-\mathrm{NMR}\left(125 \mathrm{MHz}, \mathrm{CDCl}_{3}\right) \delta$ 159.7, 146.3, 129.3, 118.8, 112.2, 112.1, 62.5, 55.2, 46.9, 34.2, 25.5. ESI-TOF calc. $\mathrm{C}_{11} \mathrm{H}_{15} \mathrm{NO}\left[\mathrm{M}+\mathrm{H}^{+}\right]$178.1226, found 178.1226.

2-(4-Chlorophenyl)pyrrolidine (6b). ${ }^{1} \mathrm{H}-\mathrm{NMR}\left(500 \mathrm{MHz}, \mathrm{CDCl}_{3}\right) \delta$ 7.30-7.25 (m, 4H), 4.09 (t, $J=7.5 \mathrm{~Hz}, 1 \mathrm{H}), 3.18$ (ddd, $J=10 \mathrm{~Hz}, 7.5 \mathrm{~Hz}, 5 \mathrm{~Hz}, 1 \mathrm{H}$ ), 3.01 (ddd, $J=10$ $\mathrm{Hz}, 8 \mathrm{~Hz}, 6.5 \mathrm{~Hz}, 1 \mathrm{H}), 2.20-2.14(\mathrm{~m}, 1 \mathrm{H}), 1.95-1.80(\mathrm{~m}, 2 \mathrm{H}), 1.65-1.57(\mathrm{~m}, 1 \mathrm{H}) .{ }^{13} \mathrm{C}-$ NMR (100 MHz, $\left.\mathrm{CDCl}_{3}\right) \delta 143.2,132.3,128.4,127.9,61.8,46.9,34.4,25.4$. ESI-TOF calc. $\mathrm{C}_{10} \mathrm{H}_{12} \mathrm{ClN}\left[\mathrm{M}+\mathrm{H}^{+}\right]$182.0731, found 182.0726.

2-(4-Bromophenyl)pyrrolidine (7b). ${ }^{1} \mathrm{H}-\mathrm{NMR}\left(500 \mathrm{MHz}, \mathrm{CDCl}_{3}\right) \delta 7.43(\mathrm{~d}, J=8 \mathrm{~Hz}$, 2H), $7.24(\mathrm{~d}, J=8.5 \mathrm{~Hz}, 2 \mathrm{H}), 4.08(\mathrm{t}, J=7.5 \mathrm{~Hz}, 1 \mathrm{H}), 3.18(\mathrm{ddd}, J=10 \mathrm{~Hz}, 7.5 \mathrm{~Hz}, 5$ Hz, 1H), 3.02 (ddd, $J=10 \mathrm{~Hz}, 8 \mathrm{~Hz}, 6.5 \mathrm{~Hz}, 1 \mathrm{H}), 2.21-2.14(\mathrm{~m}, 1 \mathrm{H}), 1.95-1.80$ (m, 2H), 
1.65-1.57 (m, 1H). ${ }^{13} \mathrm{C}-\mathrm{NMR}\left(100 \mathrm{MHz}, \mathrm{CDCl}_{3}\right) \delta$ 143.8, 131.3, 128.3, 120.3, 61.8, 46.9, 34.4, 25.4. ESI-TOF calc. $\mathrm{C}_{10} \mathrm{H}_{12} \mathrm{BrN}\left[\mathrm{M}+\mathrm{H}^{+}\right]$226.0226, found 226.0226.

2-(3-Bromophenyl)pyrrolidine (8b). ${ }^{1} \mathrm{H}-\mathrm{NMR}\left(500 \mathrm{MHz}, \mathrm{CDCl}_{3}\right) \delta 7.53(\mathrm{t}, J=1.5$ $\mathrm{Hz}, 1 \mathrm{H}), 7.36-7.34(\mathrm{~m}, 1 \mathrm{H}), 7.73-7.71(\mathrm{~m}, 1 \mathrm{H}), 7.47(\mathrm{t}, J=8 \mathrm{~Hz}, 1 \mathrm{H}), 4.08(\mathrm{t}, J=7.5 \mathrm{~Hz}$, 1H), 3.18 (ddd, $J=10 \mathrm{~Hz}, 7.5 \mathrm{~Hz}, 5 \mathrm{~Hz}, 1 \mathrm{H}), 3.02$ (ddd, $J=10 \mathrm{~Hz}, 8 \mathrm{~Hz}, 6.5 \mathrm{~Hz}, 1 \mathrm{H})$, 2.21-2.14 (m, 1H), 1.95-1.80 (m, 2H), 1.65-1.57 (m, 1H). ${ }^{13} \mathrm{C}-\mathrm{NMR}\left(150 \mathrm{MHz}, \mathrm{CDCl}_{3}\right) \delta$ $147.2,129.9,129.8,129.6,125.2,122.5,61.9,46.9,34.4,25.4$. ESI-TOF calc. $\mathrm{C}_{10} \mathrm{H}_{12} \mathrm{BrN}$ $\left[\mathrm{M}+\mathrm{H}^{+}\right]$226.0226, found 226.0228.

2-[4-(Trifluoromethyl)phenyl]pyrrolidine (9b). ${ }^{1} \mathrm{H}-\mathrm{NMR}\left(500 \mathrm{MHz}, \mathrm{CDCl}_{3}\right) \delta 7.56$ $(\mathrm{d}, J=8 \mathrm{~Hz}, 2 \mathrm{H}), 7.49(\mathrm{~d}, J=8 \mathrm{~Hz}, 2 \mathrm{H}), 4.21(\mathrm{t}, J=7.5 \mathrm{~Hz}, 1 \mathrm{H}), 3.21(\mathrm{ddd}, J=10 \mathrm{~Hz}$, $7.5 \mathrm{~Hz}, 5.5 \mathrm{~Hz}, 1 \mathrm{H}), 3.06$ (ddd, $J=10 \mathrm{~Hz}, 8 \mathrm{~Hz}, 6.5 \mathrm{~Hz}, 1 \mathrm{H}), 2.87$ (bs, 1H), 2.27-2.20 $(\mathrm{m}, 1 \mathrm{H}), 1.98-1.82(\mathrm{~m}, 2 \mathrm{H}), 1.71-1.63(\mathrm{~m}, 1 \mathrm{H}) .{ }^{13} \mathrm{C}-\mathrm{NMR}\left(125 \mathrm{MHz}, \mathrm{CDCl}_{3}\right) \delta 148.9$, 129.1, 128.8, 126.8, 125.6, $125.2(\mathrm{q}, J=3.5 \mathrm{~Hz}), 122.8,62.0,46.9,34.4,25.4$. ESI-TOF calc. $\mathrm{C}_{11} \mathrm{H}_{12} \mathrm{~F}_{3} \mathrm{~N}\left[\mathrm{M}+\mathrm{H}^{+}\right] 216.0995$, found 216.0997 .

2-(3-Nitrophenyl)pyrrolidine (10b). ${ }^{1} \mathrm{H}-\mathrm{NMR}\left(500 \mathrm{MHz}, \mathrm{CDCl}_{3}\right) \delta 8.26(\mathrm{t}, J=2 \mathrm{~Hz}$, $1 \mathrm{H}), 8.09-8.07(\mathrm{~m}, 1 \mathrm{H}), 7.73-7.71(\mathrm{~m}, 1 \mathrm{H}), 7.47(\mathrm{t}, J=8 \mathrm{~Hz}, 1 \mathrm{H}), 4.27(\mathrm{t}, J=7.5 \mathrm{~Hz}$, 1H), 3.21 (ddd, $J=10 \mathrm{~Hz}, 7.5 \mathrm{~Hz}, 5.5 \mathrm{~Hz}, 1 \mathrm{H}), 3.09$ (ddd, $J=10 \mathrm{~Hz}, 8 \mathrm{~Hz}, 6.5 \mathrm{~Hz}, 1 \mathrm{H})$, 2.30-2.23 (m, 1H), 1.99-1.83 (m, 2H), 1.70-1.63 (m, 1H). ${ }^{13} \mathrm{C}-\mathrm{NMR}\left(125 \mathrm{MHz}, \mathrm{CDCl}_{3}\right) \delta$ $147.4,132.8,129.1,122.3,121.7,121.6,61.5,46.9,34.6,25.5$. ESI-TOF calc. $\mathrm{C}_{10} \mathrm{H}_{12} \mathrm{~N}_{2} \mathrm{O}_{2}\left[\mathrm{M}+\mathrm{H}^{+}\right]$193.0971, found 193.0969. 


\section{2-(4-fluorophenyl)pyrrolidine $\quad \mathrm{CDCl} 3 \quad 500 \mathrm{MHz}$}

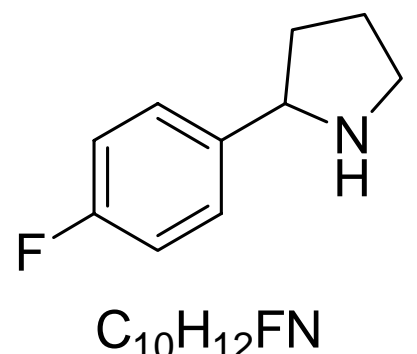

$4 b$

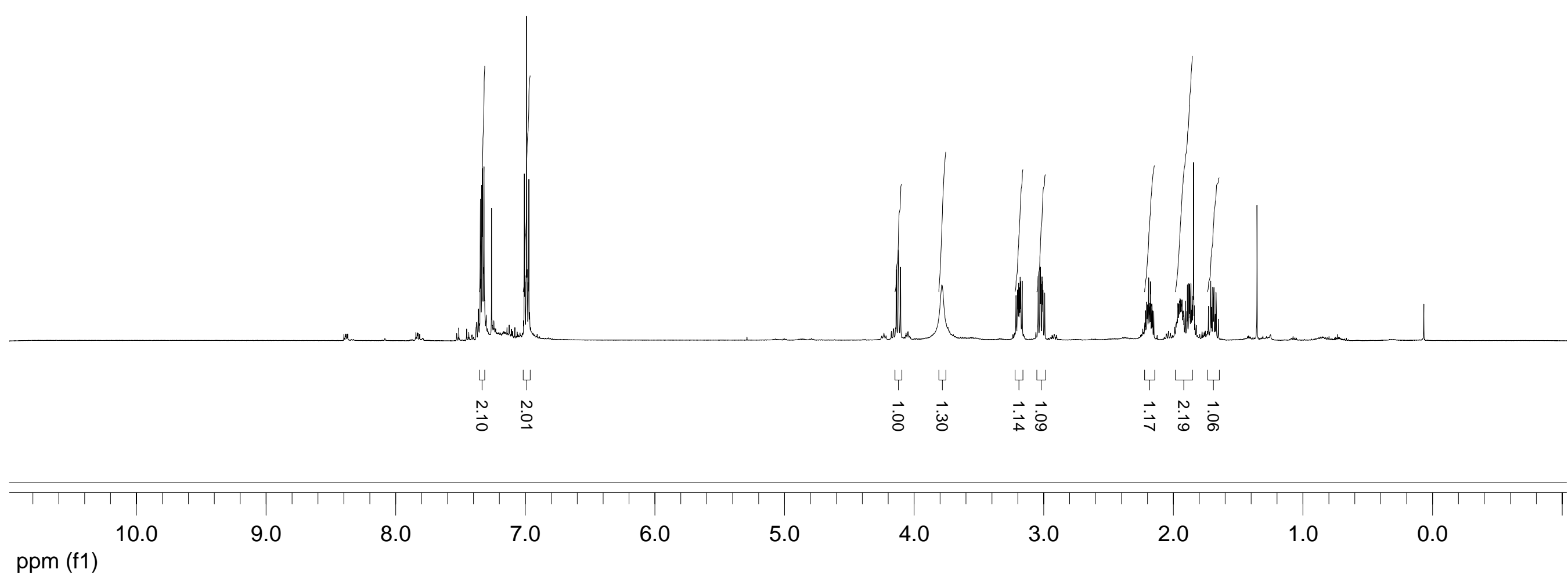


<smiles>Fc1ccc(C2CCCN2)cc1</smiles>

$\mathrm{C}_{10} \mathrm{H}_{12} \mathrm{FN}$

$4 \mathrm{~b}$

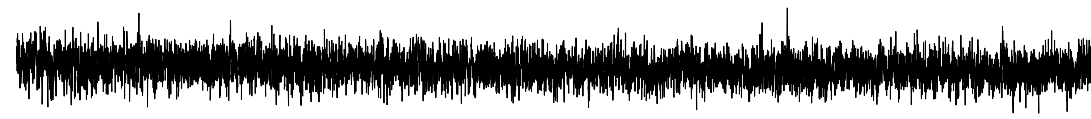

$$
\bar{\square}
$$

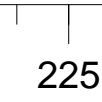
ppm (f1)
175

150

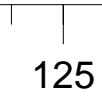

125

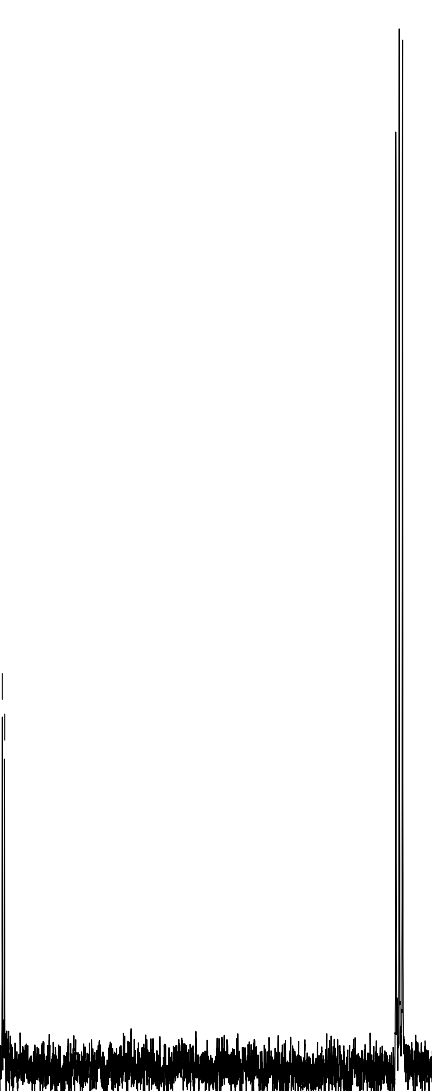

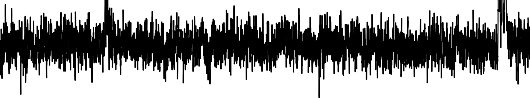

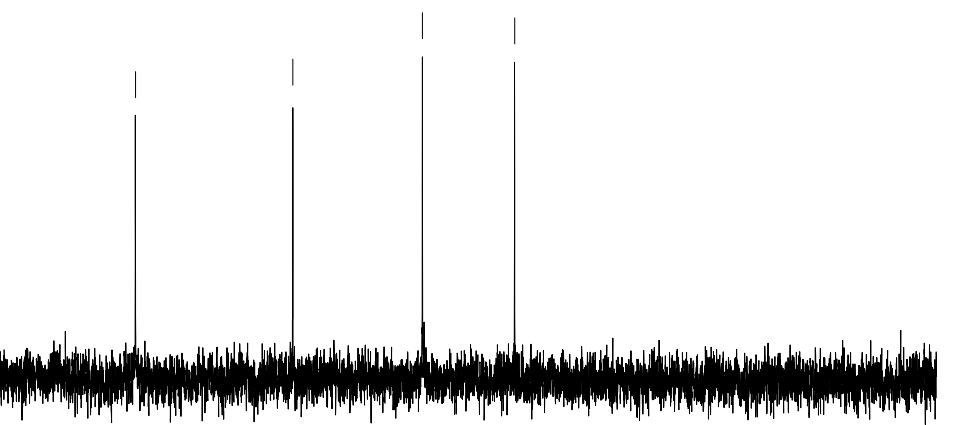

75
50
25
0 

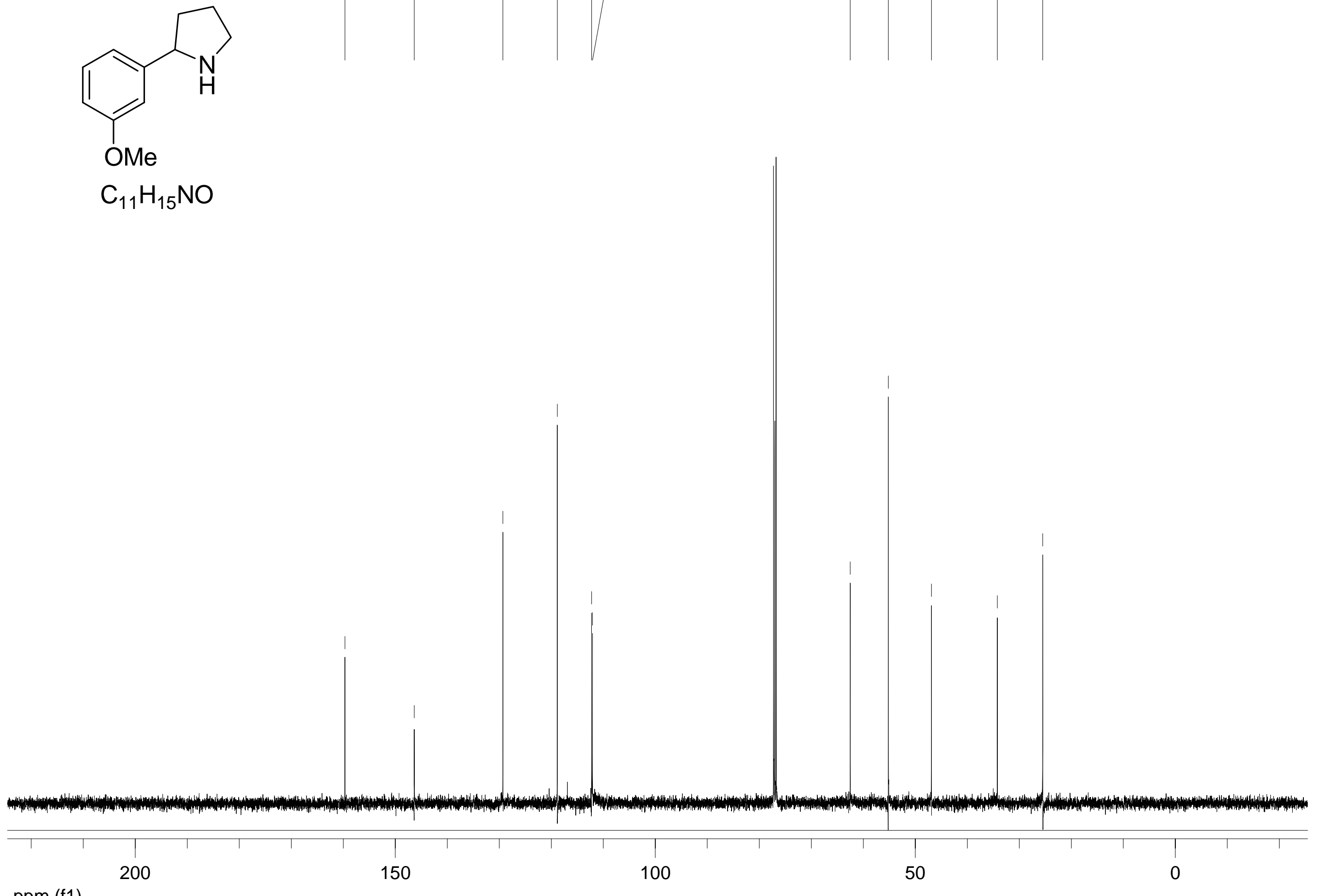


\section{2-(4-Chlorophenyl)pyrrolidine $\quad \mathrm{CDCl} 3 \quad 500 \mathrm{MHz}$}

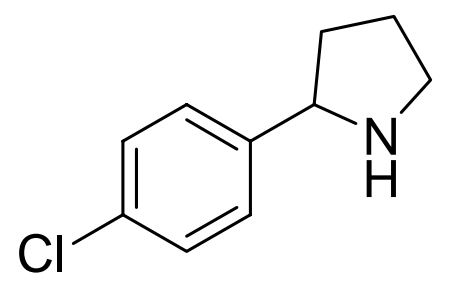

\section{$\mathrm{C}_{10} \mathrm{H}_{12} \mathrm{CIN}$}

$6 \mathrm{~b}$

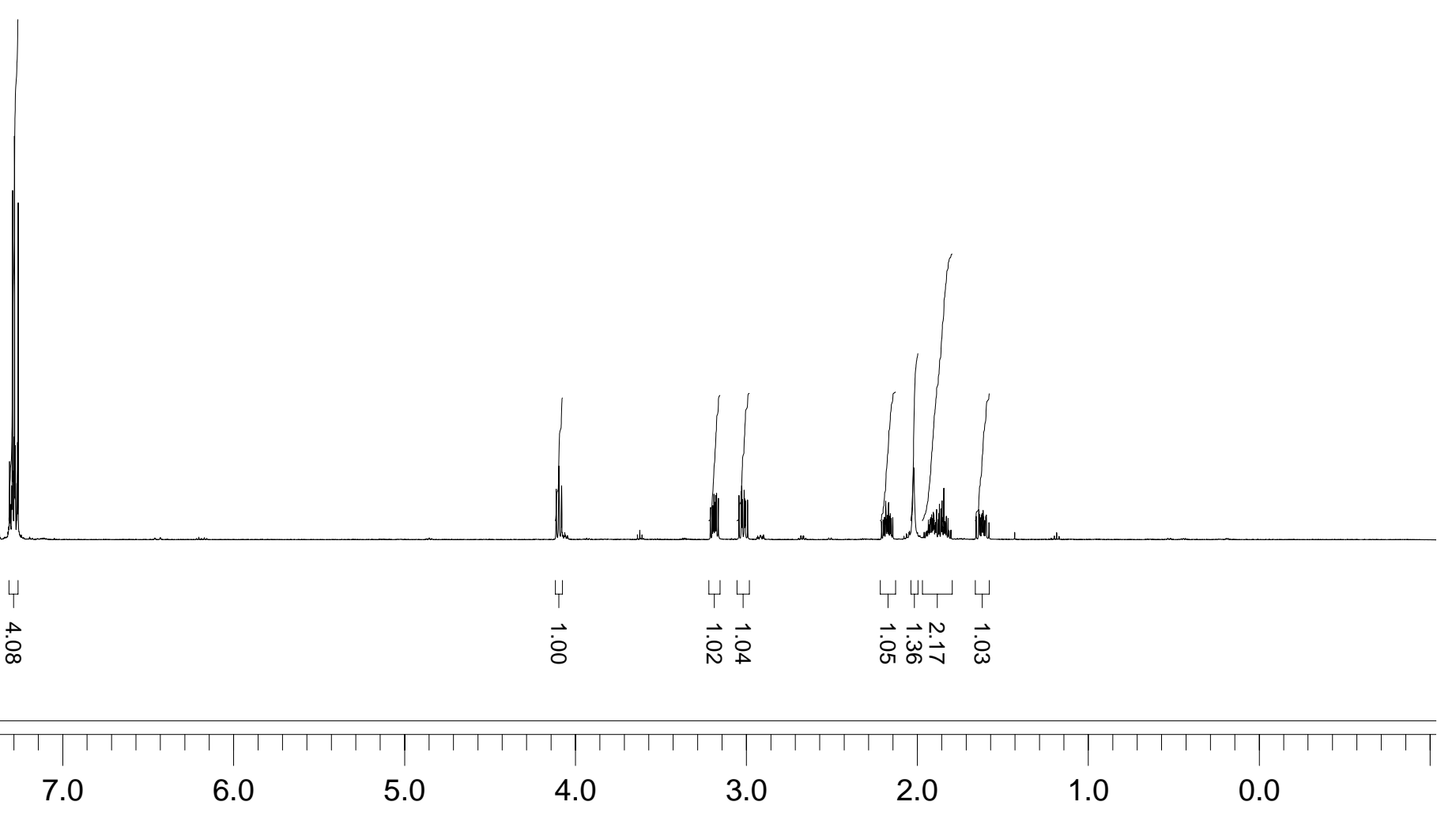




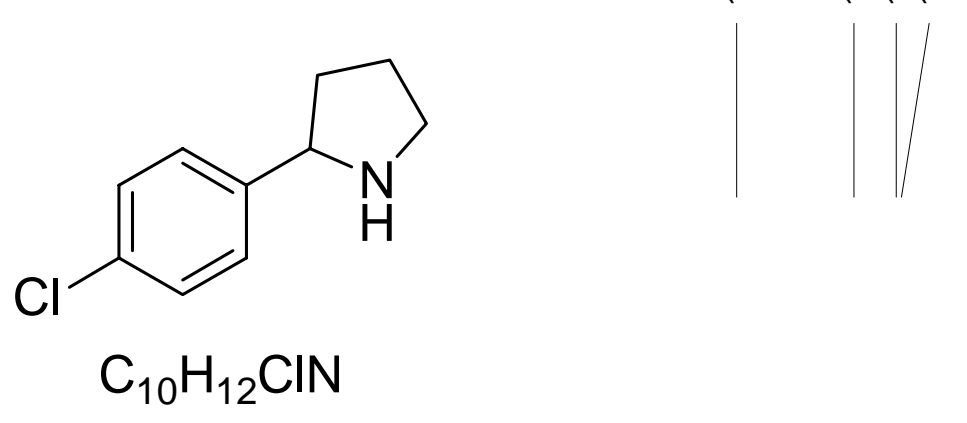

$6 b$ 


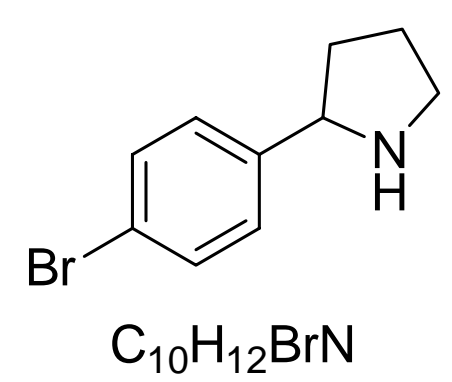

$7 b$

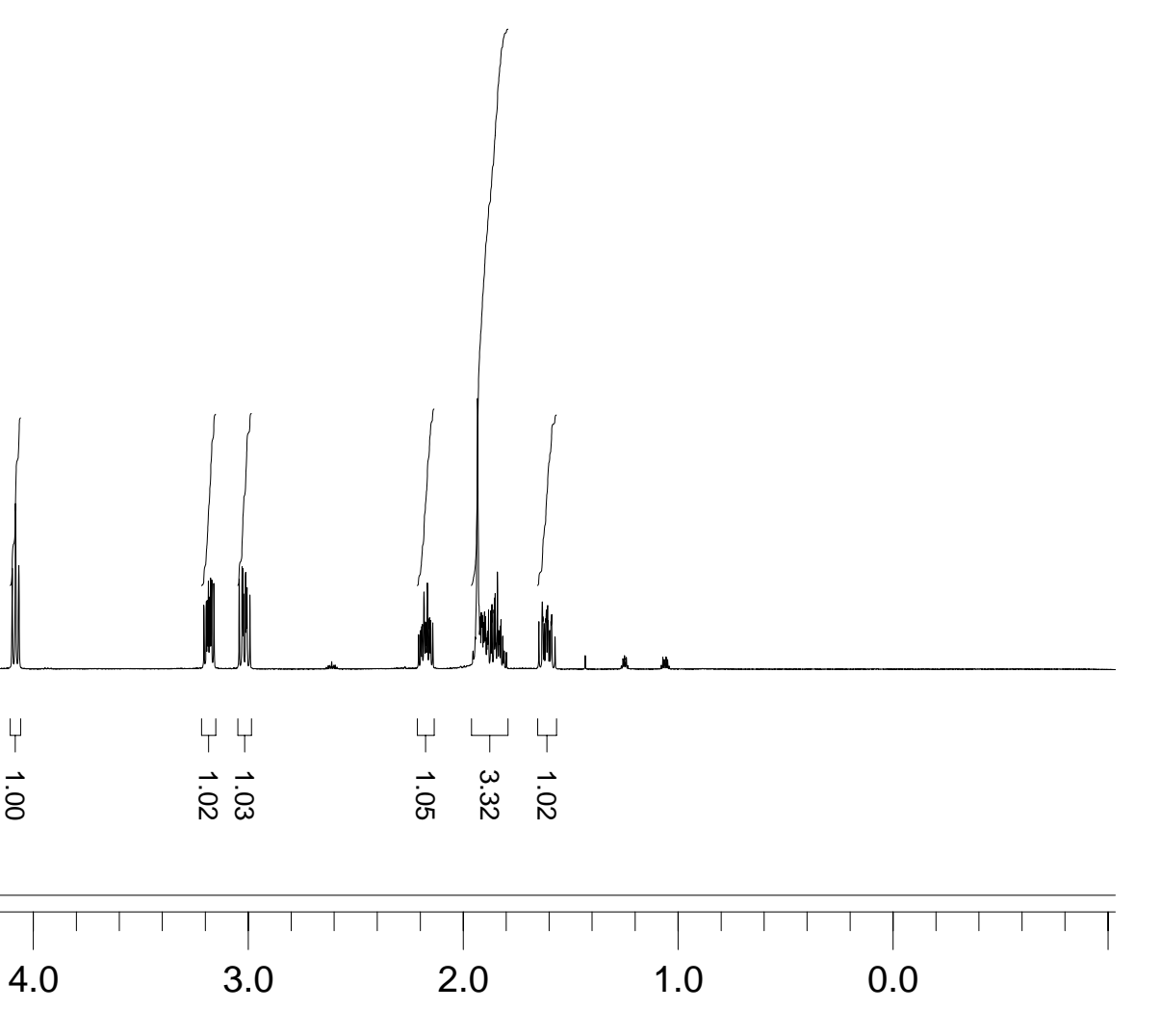

훙

9.0

8.0

7.0

${ }^{6.0} \mathrm{~S} 11$

5.0

4.0

ب

蓄 


$$
\text { 2-(4-bromophenyl)pyrrolidine CDCl3 }
$$$$
7 b
$$

. 
<smiles>Brc1cccc(C2CCCN2)c1</smiles>

$\mathrm{C}_{10} \mathrm{H}_{12} \mathrm{BrN}$

$8 b$

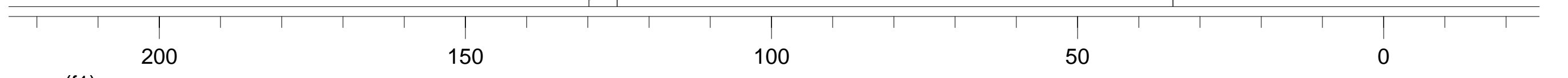




\section{$\mathrm{CDCl} 3 \quad 100 \mathrm{MHz}$}<smiles>FC(F)(F)c1ccc(C2CCCN2)cc1</smiles>

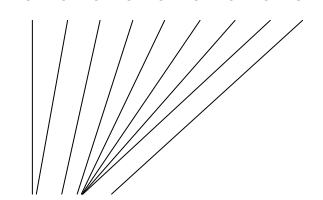

$$
\mathrm{C}_{11} \mathrm{H}_{12} \mathrm{~F}_{3} \mathrm{~N}
$$

$9 b$ 
